\title{
Optical gain in ZnCdSe-ZnSe quantum well structures
}

\author{
Paul Rees, Jon.F.Heffernan, Fred.P.Logue, John.F.Donegan, \\ Christophe Jordan, John Hegarty, Futoshi.Hiei ${ }^{*}$ and Akira Ishibashi* \\ Physics Department, Trinity College, Dublin 2, Ireland \\ "Sony Research Center, Fujitsuka-cho 174, Hodogaya-ku, Yokohama-shi 240, Japan
}

\begin{abstract}
We have measured the gain spectrum of an optically pumped $40 \AA \mathrm{ZnCdSe}-\mathrm{ZnSe}$ multiple quantum well. Our calculation, which includes many body effects such as Coulomb enhancement and spectral broadening due to carrier scattering, gives excellent agreement with the experimental gain measurements. We then show the importance of the inclusion of the Coulomb enhancement for the calculation of optical gain when predicting laser threshold currents. This is emphasised by using our gain calculation as a basis to theoretically optimise a simple $\mathrm{ZnCdSe}-\mathrm{ZnSe}$ quantum well laser structure incorporating the leakage current over the p-type cladding.
\end{abstract}

Keywords: semiconductor laser diodes, II-VI, optical gain, many body effects

\section{INTRODUCTION}

The drive for shorter wavelength light emitting devices means that research into materials such as wide band gap II-VI and GaN semiconductors is being actively pursued. Structures incorporating $\mathrm{ZnSe}-\mathrm{ZnCdSe}$ quantum wells are likely candidates for the fabrication of commercial blue-green semiconductor lasers, with devices incorporating these wells having achieved room temperature $\mathrm{cw}$ lasing at $510 \mathrm{~nm}$ with operating lifetimes of up to 4 hours ${ }^{1}$. As with the design of long wavelength III-V semiconductor lasers, computer modelling of device characteristics will be an invaluable tool for optimising performance in these structures. The basis of any laser diode model is the calculation of the material gain in the structure and before any confidence can be attained in such a model the gain calculation must be verified. The most obvious method of verification is a comparison between experimental and calculated gain spectra.

The increased exciton binding energy in II-VI semiconductors compared with III-V materials led to speculation that excitons could contribute to gain in these structures. Several groups have studied lasing in these structures and proposed different mechanisms for the excitonic contribution to gain. Kawakami et al. ${ }^{2}$ have shown that in their (MQW) samples at $77 \mathrm{~K}$ there is a well defined exciton resonance even at the carrier densities necessary for lasing; they observe stimulated emission due to exciton-phonon and exciton-exciton scattering mechanisms depending on the excitation carrier density. Ding et al. ${ }^{3}$ have presented convincing evidence to show that, at least up to $220 \mathrm{~K}$, optical gain and lasing in ( $\mathrm{ZnCd}) \mathrm{Se} / \mathrm{ZnSe} \mathrm{MQWs}$ arises from the bleaching of localised excitons in the low energy tail of the inhomogeneously broadened exciton density of states. However, in laser diodes based on $\mathrm{ZnSe}$ the room temperature gain mechanism has been shown to originate from an electron-hole plasma ${ }^{4}$.

Although gain originates from an electron-hole plasma, the strong Coulombic attraction between electrons and holes gives rise to an enhancement of the optical transition rate which has been predicted theoretically to be significant in these materials, even at room temperature, ${ }^{5,6}$. We have calculated the optical gain in a $40 \AA$ $\mathrm{Zn}_{0.72} \mathrm{Cd}_{0.28} \mathrm{Se}-\mathrm{ZnSe}$ multiple quantum well including many body effects and compared this with experimentally measured spectra. From this comparison we show the effect of the Coulomb enhancement on the gain in this structure. The consequences of this result will be discussed, with emphasis on the predicted threshold currents and the leakage current due to minority carriers escaping the quantum wells. 


\section{CALCULATION OF THE OPTICAL GAIN}

The calculation of the optical gain in a quantum well is described in detail in reference 7 . The absorption spectrum is determined from the imaginary part of the optical susceptibility, which is given by

$$
\chi(\hbar \omega)=\sum_{k} d_{k} \chi_{k}
$$

where

$$
\left[\hbar \omega-E_{e, k}-E_{h, k}+i \hbar \gamma\right] k_{k}=-\left(1-f_{e, k}-f_{h, k}\right) \times\left[d_{k}+\sum_{k^{\prime}} V_{s}\left(k-k^{\prime}\right) \chi_{k^{\prime}}\right]
$$

$f_{c}$ and $f_{v}$ are the electron and hole Fermi functions which describe the carrier distributions and $V_{s}$ is the screened two dimensional Coulomb potential within the single plasmon pole approximation ${ }^{7,8,9} \cdot d_{k}$ is the momentum dependent optical transition matrix element ${ }^{10}$ and $E_{e, k}$ and $E_{h, k}$ are the renormalised electron and hole energies at the momentum $\mathrm{k}$. The dephasing energy, $\hbar \gamma$, is calculated using Fermi's Golden rule for carrier-carrier ${ }^{11}$ and carrierLO phonon ${ }^{12}$ scattering mechanisms.

We have calculated the band structure using a $4 \mathrm{X} 4$ Luttinger-Kohn Hamiltonian including strain ${ }^{13}$. The calculations show that the bands are reasonably parabolic since the strain causes reduced intermixing of the light and heavy hole subbands. Therefore to reduce the computation time required we have used parabolic bands, the masses of which are determined using a least square fit to the calculated subband structure (this approach was used in reference 14). All material parameters for the gain calculations were taken from reference 15.

\section{EXPERIMENTAL GAIN MEASUREMENTS}

We have measured the optical gain from a sample consisting of five $40 \AA \mathrm{Zn}_{0.72} \mathrm{Cd}_{0.28}$ Se quantum wells separated by $150 \AA \mathrm{ZnSe}$ barriers and surrounded by buffer layers of undoped $\mathrm{ZnSe}$. The gain spectra were measured at room temperature using the variable stripe method described by Shaklee ${ }^{16}$. The sample was cleaved to $1.6 \mathrm{~mm}$ width and excited by a frequency-tripled Q-switched YAG laser at $355 \mathrm{~nm}$. The laser delivers $5 \mathrm{~ns}$ pulses at $20 \mathrm{~Hz}$ and the sample exhibited room temperature lasing at an excitation intensity of approximately $70 \mathrm{~kW} / \mathrm{cm}^{2}$ (for the pump length of $1600 \mu \mathrm{m}$ ). Light from the laser was focused by a cylindrical lens to a rectangular stripe on the sample. The stripe width was $80 \mu \mathrm{m}$ and the length of the stripe, perpendicular to the facets, was varied using a calibrated slit. Amplified emission from one facet was dispersed in a 2 metre spectrometer with $5 \AA$ resolution and the light was detected using an optical multi-channel analyser. For an excitation stripe length of $L$, the emission intensity is given by

$$
\mathrm{I}(\mathrm{L}) \propto \frac{\mathrm{e}^{\mathrm{g}(\hbar \omega) \mathrm{L}}-1}{\mathrm{~g}(\hbar \omega)}
$$

where $g(\hbar \omega)$ is the gain in the sample. A gain spectrum is then calculated from the solution of

$$
\frac{\mathrm{I}\left(\mathrm{L}_{1}\right)}{\mathrm{I}\left(\mathrm{L}_{2}\right)}=\frac{\left(\mathrm{e}^{\mathrm{g}(\hbar \omega) \mathrm{L}_{1}}-1\right)}{\left(\mathrm{e}^{\mathrm{g}(\hbar \omega) \mathrm{L}_{2}}-1\right)}
$$

where $L_{1}$ and $L_{2}$ are different excitation stripe lengths.

Figure 1(a) shows a typical measured gain spectrum (circles) at an excitation intensity of $80.5 \mathrm{~kW} / \mathrm{cm}^{2}$. The pump wavelength being much shorter than the bandgap of the quantum well, introduces significant carrier heating. To obtain a carrier temperature of $300 \mathrm{~K}$ the gain spectrum in figure 1 (a) was measured at a lattice temperature of $230 \mathrm{~K}$. 


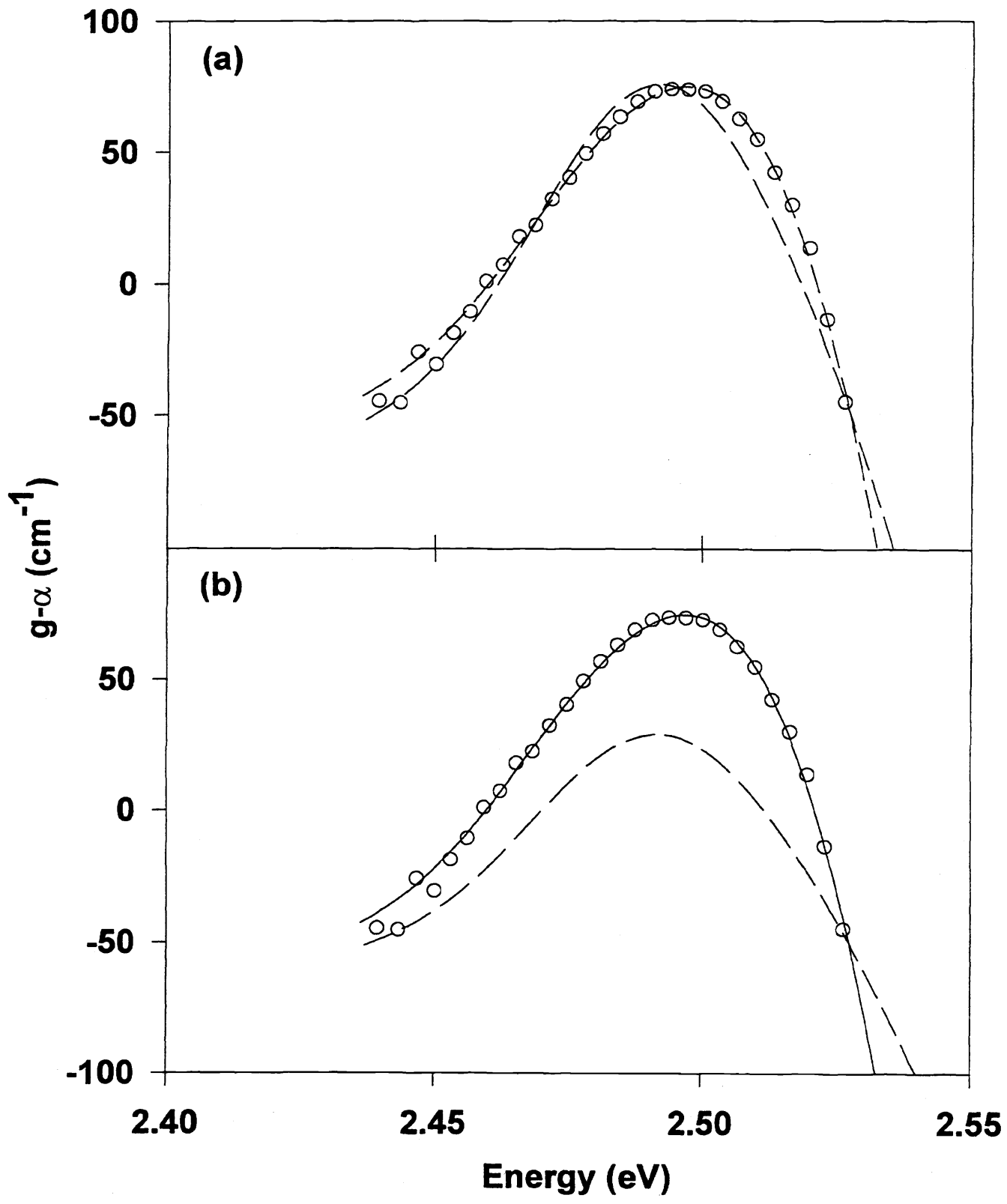

Figure 1(a). Fit to the experimentally measured gain spectrum (open circles) at a carrier temperature of $300 \mathrm{~K}$ using the many body theory and a confinement factor of 0.044 (solid line) and the free carrier theory using $a$ confinement factor of 0.07 (dashed line).

(b) As (a) but both calculated spectra scaled by the same confinement factor (0.044). 
The carrier temperature was measured from the slope of the high energy side of the front surface spontaneous emission ${ }^{17,18}$. The solid line in figure 1(a) is the gain calculated as described in section 3 . The gain measured using the procedure described above is the modal gain; the material gain, calculated by the model, was converted to the modal gain using a confinement factor of 0.04 .

\section{THE EFFECT OF COULOMB ENHANCEMENT ON THE GAIN SPECTRA}

There is excellent agreement between the experimental and theoretical gain curves in figure 1 (a). The theoretical gain was fitted to the data by varying the quasi-Fermi level separation. It should be noted that this is the only nonlinear fitting parameter in the calculation since both the carrier temperature and the material composition have been measured experimentally. Although our model includes band gap renormalisation, the absolute value of the band edge is difficult to model and the calculated spectra in figure 1(a) must be shifted rigidly in energy. This, however, does not affect the shape of the gain spectrum since band gap renormalisation is energy independent.

The high energy side of the gain spectrum is particularly sensitive to the carrier temperature and the strength of the Coulomb enhancement. The use of an experimentally determined carrier temperature in the calculation means that we are successfully predicting the magnitude of the Coulomb enhancement. The width and the shape of the gain spectra on the low energy side is dependent on the homogeneous and inhomogeneous broadening. Inhomogeneous broadening due to well width and compositional fluctuations was incorporated in the calculation as a Gaussian convolution of the individual optical transitions. The inhomogeneous linewidth was obtained from the heavy hole exciton resonance in the absorption spectrum measured at $10 \mathrm{~K}$. Again the quality of the gain prediction shows that the inclusion of the scattering calculation is a critical component of the calculation.

To date most calculations ${ }^{14,19,20}$ of the optical properties of II-VI materials have neglected the Coulombic attraction between electrons and holes. To illustrate the pronounced effect of the Coulomb enhancement on the gain we have calculated the gain neglecting the Coulomb interaction by setting the potential, $\mathrm{V}_{\mathrm{s}}$ to zero in equation 2 . The broken line in figure 1(a) shows the best fit to the data neglecting the Coulomb enhancement. For the different models to make physical sense, the transparency points at the same carrier density must coincide with the experimentally measured value. The peak energy of the gain spectrum calculated using free carrier theory does not coincide with the measured value. In fact it was impossible to account for both the peak energy and the transparency point using this theory. The effect of the Coulomb enhancement can be seen on the high energy side of the gain spectrum where the slope of the gain around the transparency point is dramatically changed. We could not account for the experimentally measured slope using a free carrier theory. In an attempt to find the best fit in figure 1(a) using the free carrier theory an increased confinement factor of 0.07 was used. If the same confinement factor is used in both calculations as is shown in figure 1(b), the significance of Coulomb enhancement is seen more clearly, the peak of the gain is increased by more than $60 \%$ by including Coulomb enhancement.

The maximum Coulomb enhancement occurs near the transparency point ${ }^{9}$ and so the peak gain does not experience the maximum enhancement However, the spontaneous emission spectrum being much broader is enhanced around it's peak, and so the spontaneous recombination current is increased to a greater extent than the maximum gain. For a given value of threshold gain the spontaneous emission is increased by the Coulomb enhancement ${ }^{6}$ leading to an increase in the threshold current density. On the other hand, Coulomb enhancement produces a larger value of gain for a smaller separation in the quasi-Fermi levels and so reduces carrier leakage. It has been shown ${ }^{21}$ that the reduction of carrier leakage significantly decreases the threshold current of II-VI diode lasers at room temperature. Such competitive effects show that a thorough understanding of Coulomb enhancement is essential for the development and optimisation of blue-green optoelectronic devices.

\section{CALCULATION OF THE LEAKAGE CURRENT}

$\mathrm{Wu}^{15}$ has carried out a comprehensive theoretical study of the leakage current in $\mathrm{ZnCdSe}-\mathrm{ZnSe}$ quantum well lasers, showing the wellwidth dependence of the threshold current and has derived an optimum structure to minimise the operating current. In this paper we calculate the leakage component of the threshold current to investigate the effect of the Coulomb enhancement on such an analysis. 
The leakage current calculation follows those reported in references 15 and 22 . The electron leakage current density, $\mathrm{J}_{\text {leakage }}$ is the sum of drift and diffusion components and is given by

$$
J_{\text {leakage }}=e_{n} N_{p}\left[\sqrt{\frac{1}{L_{n}^{2}}+\frac{1}{4 z^{2}}} \operatorname{coth}\left(\sqrt{\frac{1}{L_{n}^{2}}+\frac{1}{4 z^{2}}} w\right)+\frac{1}{2 z}\right]
$$

where $e$ is the electronic charge, $w$ is the p-cladding layer thickness and $N_{p}$ is the electron population at the cladding interface. $D_{n}$ is the minority diffusion coefficient and $L_{n}$ the electron diffusion length is given by $\left(D_{n} \tau_{n}\right)^{1 / 2}$ where $\tau_{n}$ is the minority carrier lifetime. $z$ is a length characteristic of drift leakage and is given by

$$
z=\left(\frac{k_{B} T}{e}\right) \frac{\sigma_{p}}{J_{\text {total }}}
$$

where $\sigma_{\mathrm{p}}$ is the electrical conductivity of the p-cladding, $T$ the electron temperature and $\mathrm{J}_{\text {total }}$ is the total diode current density.

To illustrate the effect of the inclusion of the Coulomb enhancement on the determination of the threshold current, we have calculated the threshold current density of a $\mathrm{Zn}_{0.72} \mathrm{Cd}_{0.28} \mathrm{Se}-\mathrm{ZnSe}$ single quantum well laser. We have assumed a cladding layer of $\mathrm{ZnMgSSe}$ which giving an electron energy offset of $50 \mathrm{meV}$ over the $\mathrm{ZnSe}$ barrier (width of $70 \mathrm{~nm}$ ). For our example we use a laser cavity length of $330 \mu \mathrm{m}$ and assume an internal scattering loss of $30 \mathrm{~cm}^{-1}$. Again, all material parameters used to calculate the leakage current are taken from reference 15 .

Figure 2 shows the threshold current density of the single quantum well laser structure as a function of well width at a temperature of $300 \mathrm{~K}$. The solid and broken lines correspond to the current densities calculated with and without the Coulomb enhancement, respectively. The solid circles and triangles represent the current densities calculated with and without the inclusion of the leakage. The threshold current density without the leakage component is simply the spontaneous recombination current multiplied by the internal quantum efficiency, which we assume to be $100 \%$ for this example. All other losses such as Auger recombination are assumed to be negligible and we also ignore the radiative recombination of carriers in the barrier. Well width fluctuations are included as a Gaussian distribution about the nominal width with a standard deviation of 1 monolayer ${ }^{23}$.

At large well widths the threshold current density is dominated by the spontaneous recombination current, the leakage current is smaller in wide wells because the quantum confined energy levels in the well are deeper and so the thermal activation of the carriers is minimised. It is clear that the Coulomb enhancement increases the spontaneous recombination current density required to achieve the threshold gain for all values of well width. The slight increase in spontaneous recombination current at narrower well widths is due to the inhomogenous broadening due to well width fluctuations which become increasingly more important as the well width decreases. This inhomogeneous broadening appears to be smaller in the $\mathrm{ZnCdSe}-\mathrm{ZnSe}$ material system than for example in $\mathrm{GaInP}^{23}$, due to the higher effective masses of both the electrons and holes, which means the lower energy levels in the quantum wells are less dependent on the well width.

As the well width decreases the leakage current increases due to the increased quantum confinement raising the energy levels in the well, increasing the thermally activated carrier leakage. The Coulomb enhancement increases the amount of gain obtained at a fixed Fermi-level separation, thus the gain required to overcome the cavity losses can be obtained at a smaller Fermi-level separation. The leakage current density predicted is then higher when the Coulomb enhancement is ignored due to the conduction Fermi level being driven higher into the electron subband to achieve the same threshold value of gain. This leads to the situation where the leakage current becomes dominant at narrower well widths than when the Coulomb enhancement is ignored. This means we can move to narrower well widths to take advantage of the decreased spontaneous recombination current due to increased quantum confinement without the leakage current starting to dominate. As can be seen from figure 2, 


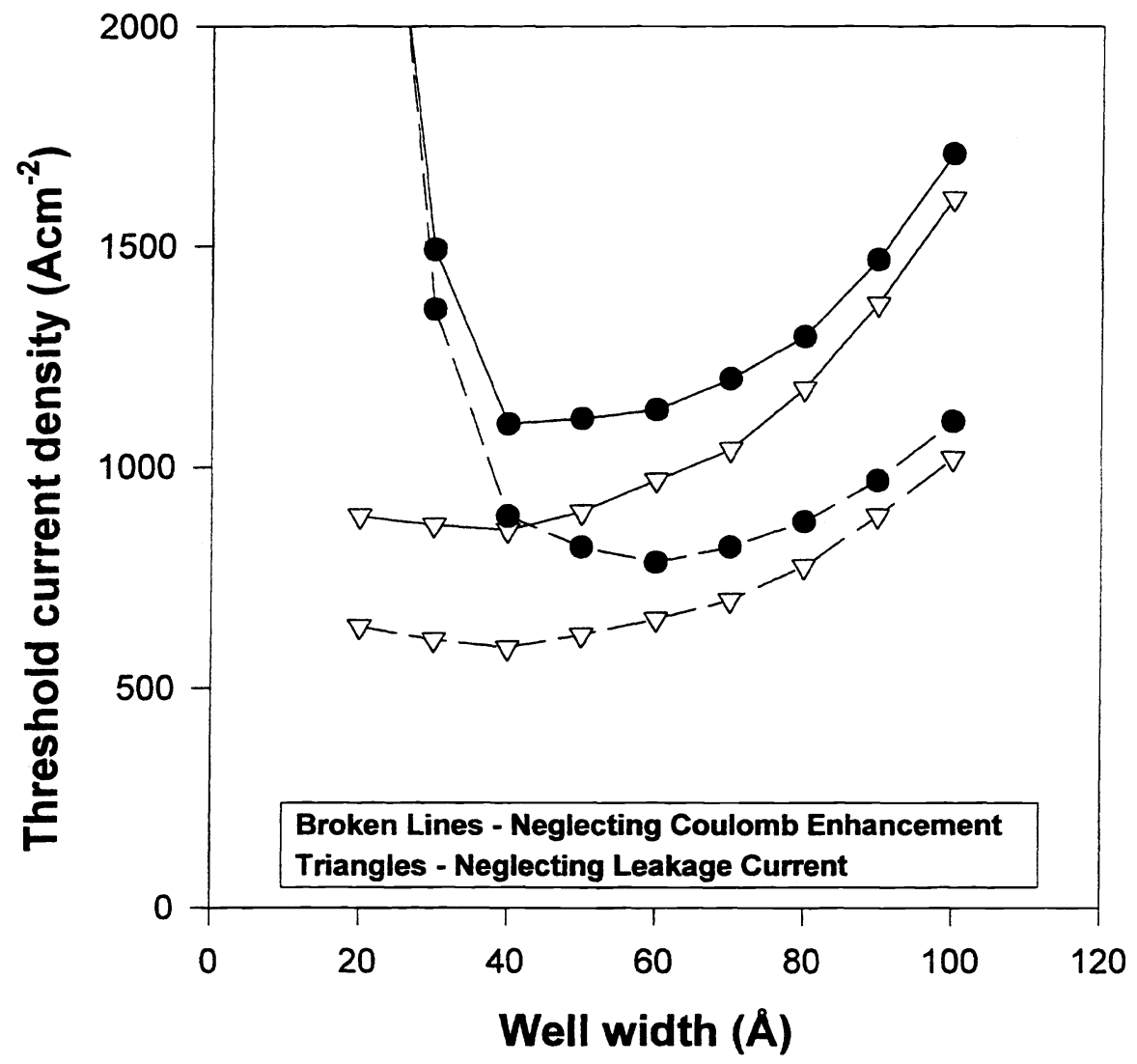

Figure 2. The calculated threshold current density of a $\mathrm{Zn}_{0.72} \mathrm{Cd}_{0.28} \mathrm{Se}-\mathrm{ZnSe}$ quantum well laser as a function of well width.

in our example this leads to the situation where the minimum threshold current density is achieved with a well width of $40 \AA$, although when the Coulomb enhancement is ignored the optimum well width predicted is $60 \AA$. It is clear that Coulomb enhancement must be included in the calculation of the gain-current relationship incorporated in any model employed to optimise the device structure of a $\mathrm{ZnCdSe}-\mathrm{ZnSe}$ quantum well laser. This would be especially important when predicting the threshold current dependence on the temperature of these devices due to the increased leakage current at the higher temperatures.

\section{CONCLUSIONS .}

In conclusion, we have measured the optical gain from a sample consisting of five $40 \AA \mathrm{Zn}_{0.72} \mathrm{Cd}_{0.28} \mathrm{Se}$ quantum wells. The gain from an electron-hole plasma was calculated including many body effects and was shown to be in excellent agreement with experiment. From this comparison we have shown the effect of the Coulomb interaction to be a very important consideration in the calculation of the gain spectra for $\mathrm{ZnCdSe}-\mathrm{ZnSe}$ structures. The consequences of the Coulomb enhancement on the calculated threshold current density of a laser diode were also discussed. We show there is a discrepancy in the conduction quasi Fermi-level when Coulomb effects are 
neglected and that the subsequent calculation of thermally activated leakage current can be underestimated. This is highlighted by a simple calculation of the optimum well width of a $\mathrm{Zn}_{0.72} \mathrm{Cd}_{0.28}$ Se single quantum well laser.

\section{ACKNOWLEDGEMENTS}

P.Rees is funded by the EC under the Human Capital Mobility research training programme. The authors would like to thank Ian Galbraith, Peter Smowton and Craig Cooper for their help and advice.

\section{REFERENCES}

1. A.Ishibashi, private communication

2. Y.Kawakami, I.Hauksson, H.Stewart, J.Simpson, I.Galbraith, K.A.Prior and B.C.Cavenett, "Exciton-related lasing mechanism in ZnSe-ZnCdSe mutiple quantum wells", Phys.Rev. B48, pp1 1994-12000 (1993)

3. J.Ding, H.Jeon, T.Ishihara, M.Hagerott, A.V.Nurmikko, H.Luo, N.Samarth and J.Furdyna, "Excitonic gain and laser emission in ZnSe based quantum wells", Phys.Rev.Lett. 69, pp1707-1710 (1992)

4. H.Okuyama, E.Kato, S.Itoh, N.Nakayama, T.Ohata and A.Ishibashi, "Operation and dynamics of ZnSe/ZnMgSSe double heterostructure blue laser diode at room temperature", Appl.Phys.Letts. 66, (6), pp656-658 (1995)

5. W.W.Chow and S.W.Koch, "Many-body Coulomb effects in room temperature II-VI quantum well semiconductor lasers", Appl.Phys.Lett. 66, pp3004-3006 (1995)

6. P.Rees, F.P.Logue, J.F.Donegan, J.F.Heffernan, C.Jordan and J.Hegarty "Calculation of gain-current characteristics in $\mathrm{ZnCdSe}-\mathrm{ZnSe}$ quantum well structures including many body effects", Appl.Phys.Letts. (to be published 18th Dec 95)

7. H.Haug and S.W.Koch, "Quantum theory of the optical and electronic properties of semiconductors", World Scientific, Singapore 1990

8. M.J.Snelling, P.Perozzo, D.C.Hutchings, I.Galbraith and A.Miller, "Investigation of excitonic saturation by timeresolved circular dichroism in GaAs-AlGaAs multiple quantum wells", Phys.Rev B49, pp 17160-17169 (1994)

9. H.Haug and S.W.Koch, "Semiconductor laser theory with many body effects", Phys.Rev. A39, pp1887-1898 (1989)

10. E.Kane, "Thomas-Fermi approach to impure semiconductor band structure", Phys.Rev. 131, pp79-88 (1963)

11. R.A.H.Hamilton and P.Rees, "Line broadening due to carrier-carrier scattering in quantum well heterostructures", Semicond.Sci.Tech. 8, pp728-734 (1993)

12. M.Asada, "Intrband relaxation time in quantum-well lasers", IEEE J.Quant.Electron. 25, pp2019-2026 (1989)

13. S.L.Chuang, "Efficient band-structure calculations of strained quantum wells Phys.Rev. B43, pp9649-9653 (1991)

14. P.M.Mensz, "Prospect for truly blue $\mathrm{ZnSe} / \mathrm{ZnMgSSe} / \mathrm{ZnMgSSe}$ semiconductor diode lasers", Appl.Phys.Lett. 65, pp2627-2629 (1994)

15. Y-H.Wu, "Structure-dependent threshold current density for CdZnSe-based II-VI semiconductor lasers", IEEE J.Quant.Electron. 30, pp1562-1573 (1994)

16. K.L.Shaklee, R.E.Nahory and R.F.Leheny, "Optical gain in semiconductors", J.Lumin. 7, pp284-309 (1973)

17. E.S-M.Tsui, P.Blood and E.D.Fletcher, "Electroluminescent processes in quantum well structures", Semicond.Sci.Technol. 7, pp837-844 (1992)

18. W.S.Pelouch, R.J.Ellingson, P.E.Powers, C.L.Tang, D.M.Szmyd and A.J.Nozik, "Comparison of hot-carrier relaxation in quantum wells and bulk GaAs at high carrier densities", Phys.Rev. B45, pp1450-1453 (1992)

19. R.L.Aggarwal, J.J.Zayhowski and B.Lax, "Calculated room temperature threshold current densities for the visible II-VI ZnCdSe/ZnSe quantum well laser diodes", Appl.Phys.Lett. 62, pp2899-2901, (1993)

20. D.Ahn, T-K.Koo and H.Y.Lee, "Optical gain of CdZnSe/ZnSe quantum well lasers", Appl.Phys.Lett. 59, pp2669-2671 (1991).

21. M.Buijs, K.Shahzad, S.Flamholtz, K.Haberern and J.Gaines, "Carrier leakage in blue-green II-VI lasers", Appl.Phys.Lett. 67, pp1987-1989 (1995)

22. G.P.Agrawal and N.K Dutta, 'Long wavelength semiconductor lasers', Van Nostrand Reinhold, New York (1986)

23 P.M.Smowton, H.D.Summers, P.Rees and P.Blood, IEEE Photonics Technology Letters 6, pp910-912 (1994) 